\title{
Real-Time 3D Curved Needle Segmentation Using Combined B-Mode and Power Doppler Ultrasound
}

\author{
Joseph D. Greer, Troy K. Adebar, Gloria L. Hwang, and Allison M. Okamura \\ Stanford University, Stanford, CA, USA
}

\begin{abstract}
This paper presents a real-time segmentation method for curved needles in biological tissue based on analysis of B-mode and power Doppler images from a tracked 2D ultrasound transducer. Mechanical vibration induced by an external voice coil results in a Doppler response along the needle shaft, which is centered around the needle section in the ultrasound image. First, B-mode image analysis is performed within regions of interest indicated by the Doppler response to create a segmentation of the needle section in the ultrasound image. Next, each needle section is decomposed into a sequence of points and transformed into a global coordinate system using the tracked transducer pose. Finally, the $3 \mathrm{D}$ shape is reconstructed from these points. The results of this method differ from manual segmentation by $0.71 \pm 0.55 \mathrm{~mm}$ in needle tip location and $0.38 \pm 0.27 \mathrm{~mm}$ along the needle shaft. This method is also fast, taking 5-10 ms to run on a standard PC, and is particularly advantageous in robotic needle steering, which involves thin, curved needles with poor echogenicity.
\end{abstract}

\section{Introduction}

Needle-based percutaneous diagnostic and interventional procedures allow access to anatomical targets deep inside the body with minimal trauma to the patient. In many of these procedures, needles are inserted using image guidance by real-time ultrasound. While ultrasound has many advantages - it is fast, safe, portable, and inexpensive - standard B-mode (grayscale) ultrasound generally produces images with poor needle visibility [3]. This can make it difficult for clinicians to localize the needle with respect to anatomy. Poor needle visibility is also problematic in image-guided robotic interventions, which require automatic segmentation of needles from image data. A needle steered by a robot requires segmentation techniques that can accurately identify the shape of very thin needles inserted along curved paths through biological tissue. This image analysis problem motivates the segmentation method described in this paper.

Most existing methods for ultrasound segmentation apply peak detection on the Hough or Radon transform of the image. Offline methods have been described for segmenting curved needles by detection of a sequence of straight line segments that are combined to form a curved result [112, as well as a Hough transform

P. Golland et al. (Eds.): MICCAI 2014, Part II, LNCS 8674, pp. 381-388, 2014.

(C) Springer International Publishing Switzerland 2014 
modified to include needle bending parameters directly 11/214. Real-time methods have been demonstrated for segmentation of straight needles. These include dual-plane projections [4] and coarse-fine sampling [515]. All of these methods assume that the needle is the brightest reflector in the ultrasound data, and also that there are no roughly linear objects other than the needle. These may not be valid assumptions in biological tissue.

The underlying issues that make automatic needle segmentation difficult in biological tissue are twofold: the poor visibility of needles in B-Mode ultrasound, and the visual similarity of needles to biological structures such as vessels and tissue boundaries. Coatings or surface treatments can improve the echogenicity of needles [3], but computationally intensive methods based on the Hough transform are still necessary to isolate the needle from the surrounding anatomy. Ultrasound sensors mounted directly on the needle tip have been used for tracking 10. However, the flexibility and small diameter of steerable needles [2], precludes the use of mounted sensors in our work. An alternative approach is to vibrate the needle and detect the motion using Doppler ultrasound. Several studies demonstrated that the resulting Doppler response can increase needle visibility and enable needle tip localization to within several millimeters [6/7/9]. Segmentation of vibrating steerable needles from Doppler data alone has also been demonstrated, with an average error of several millimeters [2].

Doppler and B-mode imaging each have advantages with regards to needle segmentation. The irregular Doppler response around a vibrating needle indicates the approximate shape and location of the needle section, while B-mode data shows a more precise, but less echogenic, response. We propose a new segmentation method, which fuses B-mode and Doppler ultrasound to combine the benefits of both modalities. This method can isolate needles from other linear structures, and is computationally simple.

Although needle vibration and Doppler imaging may be used to segment a needle that is contained entirely within the ultrasound image plane (i.e. segmentation from 2D ultrasound), this work addresses the segmentation of curved needles in non-planar configurations which arise in our motivating application, needle steering. In particular, our segmentation method uses a tracked freehand 2D transducer swept over the length of curved steerable needles in ex vivo biological tissue.

\section{Methods}

Figure 1 provides an overview of our segmentation method, in which external high-frequency vibration of the needle causes a Doppler response in the ultrasound image. This response roughly indicates the needle section's location and shape, simplifying B-mode image analysis. Our method consists of two main steps: image analysis and curve-fitting. Image analysis is applied to pairs of power Doppler and B-mode ultrasound images, in order to generate candidate needle points. Curve-fitting generates an estimate of needle shape and length based on these points. 

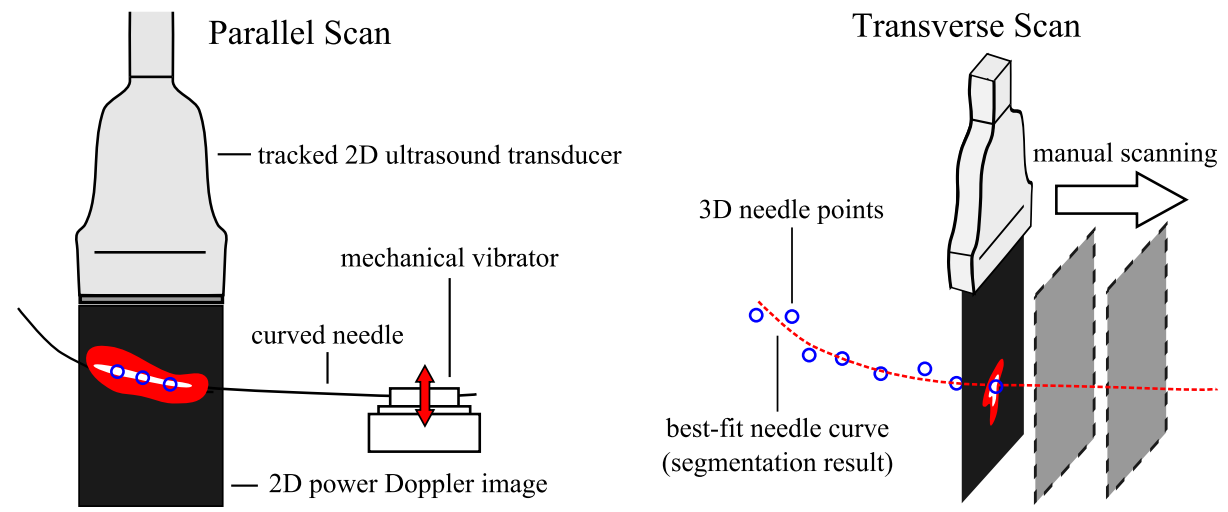

Fig. 1. Two extremes of relative needle-transducer orientation are demonstrated. A magnetically tracked freehand ultrasound transducer is manually swept over an externally vibrated, curved, steerable needle. Power Doppler and B-mode images are analyzed to generate candidate needle points (blue circles). Using the candidate needle positions, the needle shape is reconstructed in a curve-fitting step. On the left, the needle is in the ultrasound image plane. On the right, the needle appears as a point.

\subsection{Image Analysis}

Our image analysis method (Figure 2) is applied to pairs of Power Doppler and B-mode images. For each pair, the Power Doppler image is processed first, in order to identify regions of interest (ROIs) in the B-mode image. These ROIs in the B-mode image are then processed to identify candidate needle locations.

Doppler Analysis. Rectangular Doppler ROIs $\left\{R_{1}, \ldots, R_{n}\right\}$ are initially defined as bounding rectangles for the connected components of the power Doppler image. First, ROIs with area or cumulative intensity less than specified thresholds are labeled Doppler noise and discarded. These thresholds were set empirically. The ROIs are then clustered based on mutual proximity. ROIs $R_{i}, R_{j}$ are combined if $d\left(R_{i}, R_{j}\right)<\frac{1}{16} \max \left(w\left(R_{i}\right)+h\left(R_{i}\right), w\left(R_{j}\right)+h\left(R_{j}\right)\right)$ where functions $w(R)$ and $h(R)$ return the width and height of rectangular ROIs, and $d\left(R_{i}, R_{j}\right)=\min \left(\left\{\left|\boldsymbol{x}_{\boldsymbol{i}}-\boldsymbol{x}_{\boldsymbol{j}}\right|: \boldsymbol{x}_{\boldsymbol{i}} \in R_{i}, \boldsymbol{x}_{\boldsymbol{j}} \in R_{j}\right\}\right)$.

B-mode Analysis. The clustered Doppler ROIs define the portions of the Bmode image that will be analyzed. Within each ROI, the B-mode is binarized using Otsu threshold selection [13, yielding a set of connected components in the B-mode data. These connected components, or blobs, represent salient structures within the B-mode image that are in the vicinity of a Doppler response. If the needle was constrained to be nearly perpendicular to the imaging plane, these blobs would approximate circular needle cross sections, so the blob centroids could be used directly as candidate needle positions. However, since the needle may partially lie within the imaging plane, some images contain curved needle sections, which should result in multiple candidate needle points. To handle both cases, we 


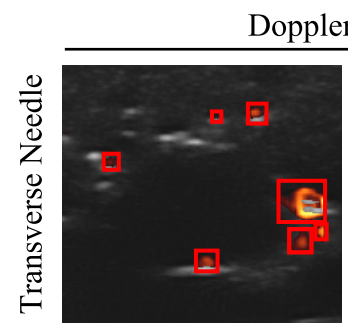

ROI Generation

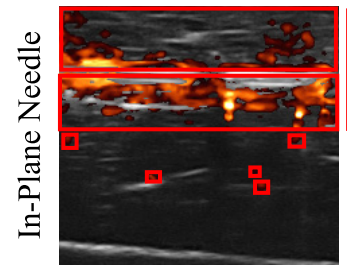

B-mode Analysis

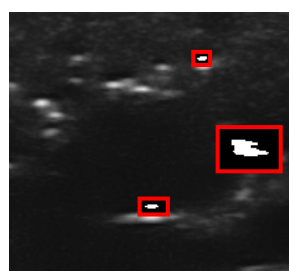

Adaptive Thresholding

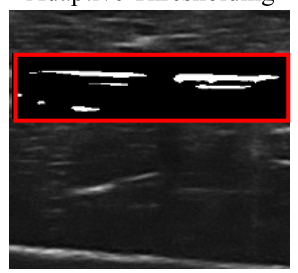

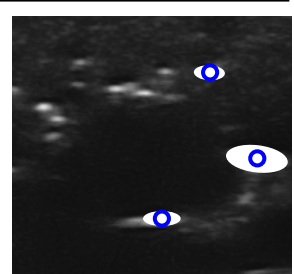

Ellipse Fit

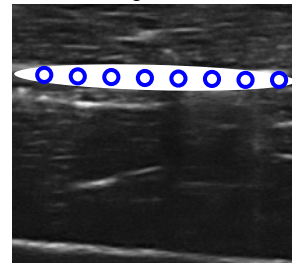

Fig. 2. Image analysis for transverse and in-plane needle sections. Regions of interest (ROIs) are generated based on the Doppler response. ROIs are clustered, filtered, and applied to the corresponding B-mode image. An adaptive threshold identifies salient features in the B-mode ROIs, and ellipses are fit to the B-mode response. One or more candidate needle points (blue circles) are identified for each ellipse, with multiple points at regular spacing for more eccentric ellipses.

assume that any in-plane needle section is small enough that it can be approximated as linear. The method determines the extent of the in-plane needle section by fitting an ellipse to each B-mode blob. It does this by modeling the B-mode intensity within each ROI as a bi-variate normal distribution with mean $\boldsymbol{\mu} \in \mathbb{R}^{2}$ and covariance $\boldsymbol{\Sigma} \in \mathbb{R}^{2 \times 2}$. The ellipse's major axis is defined by the dominant eigenvector of $\boldsymbol{\Sigma}$. As shown in Figure 2, the major axis of each ellipse is split into increments of length $\delta$, with a candidate needle position reported at the boundary of each increment. Here, we set $\delta=1.5 \mathrm{~mm}$ (three times the needle diameter) to account for the needle reverberation artifact. The $2 \mathrm{D}$ candidate needle positions are transformed to 3D world coordinates based on the recorded ultrasound transducer pose. This method does not handle highly curved needles contained within the ultrasound image, but could be extended by replacing ellipse-fitting with a step that approximates the curved shape of each B-mode blob.

Confidence Scoring. Each candidate needle point defined by image analysis is given a confidence score $s$, which represents agreement between Doppler and Bmode image data. This score is proportional to both the intensity of the Doppler response and B-mode responses, and inversely proportional to the distance between the candidate point location and the Doppler centroid.

\subsection{Curve Fitting}

At each time step (i.e., after an image pair is processed to yield new candidate needle positions), a series of curve-fitting operations update a previous model for the needle shape, defined by a vector of third-order polynomial functions 


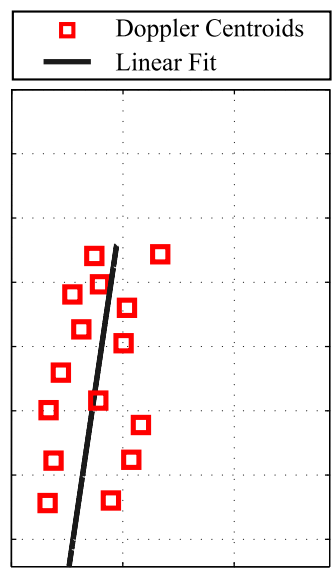

(a)
O B-mode Inliers $\quad$ Polynomial Fit

$\diamond \quad B-$ mode Outliers

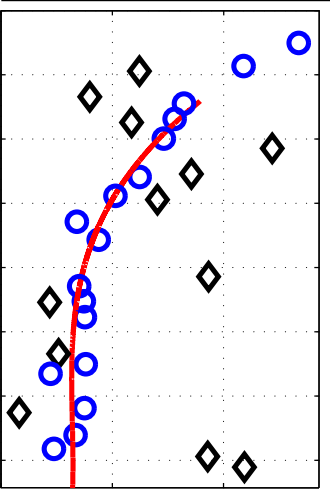

(b)

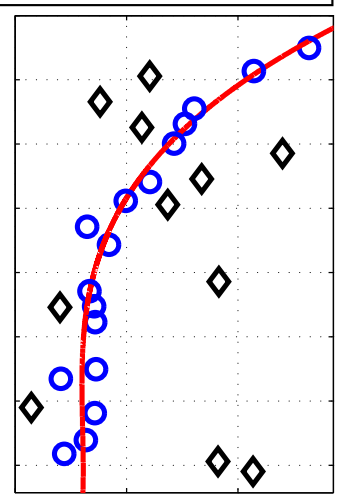

(c)

Fig. 3. Curve fitting: (a) The curve is initialized based on a linear fit to the first 30 Doppler image centroids. (b) After each image is processed, outlier candidate points are rejected based on distance from the previous curve estimate, and the curve is updated. (c) The range of the curve is updated to identify the tip.

$P=\left[p_{x}(t), p_{y}(t), p_{z}(t)\right]^{\mathrm{T}}$, and a valid parameter range $R=\left[t_{\text {base }}, t_{\mathrm{tip}}\right]^{\mathrm{T}}$. To initialize this iterative process, the needle shape is defined by a linear fit to the overall image centroids from the first 30 power Doppler frames.

Polynomial Update. Although the power Doppler response tends to be focused around the vibrating needle, Doppler responses also appear around other bright, salient features in the B-mode. Since the image analysis step erroneously labels these points as candidate needle locations, outlier rejection is a crucial component of our curve-fitting step.

At each time step, all candidate needle points from all time steps are reassessed for outlier status. A candidate needle point is labeled an outlier if its distance from the needle model generated in the previous time step is greater than an adaptive threshold, $\alpha$. As the number of measurements grows, the method becomes more selective in its labeling of inliers to increase accuracy. To accomplish this, the distance threshold, $\alpha$, starts at $5 \mathrm{~mm}$ and is decreased linearly to $0.5 \mathrm{~mm}$ as the inlier set grows to a predefined saturation point, $N_{\max }$. $\left(N_{\max }=300 \mathrm{in}\right.$ our implementation.) Using an adaptive threshold allows our method to begin with a relaxed labeling of inliers (high $\alpha$ ) to prevent convergence onto a false solution without decreasing accuracy at later time steps.

After outliers are rejected, the inliers are parameterized by their projection onto the principal axis of the set, yielding parameters $\left\{t_{1}, \ldots, t_{N_{\text {inliers }}}\right\}$. A new polynomial model $P=\left[p_{x}, p_{y}, p_{z}\right]^{\mathrm{T}}$ is then defined by a least-squares fit to the $x-y-z$ coordinates of each of the inlier points, weighted by the set of image confidence scores $\left\{s_{1}, \ldots, s_{N_{\text {inliers }}}\right\}$. 
Range Update. The parameter range $R=\left[t_{\text {base }}, t_{\mathrm{tip}}\right]^{\mathrm{T}}$ defines the location of the needle tip along the polynomial curve. In many needle segmentation methods, locating the needle tip along the needle axis is the most difficult step because noise or poor echogenicity of the needle may cause the tip estimate to be grossly incorrect. In our case, simply updating the parameter range based on the new range, $\left[t_{1}, t_{N_{\text {inliers }}}\right]$, can result in large errors, due to spurious Doppler response far from the needle. Instead, for $t_{k}>t_{\text {tip }}, t_{\text {tip }}$ is updated to $t_{k}$ if and only if $\frac{1}{\left(t_{k}-t_{\text {tip }}\right)^{2}} \sum_{s_{i} \in \mathcal{I}_{t_{\text {tip }}}^{t_{k}}} s_{i}>\sigma$, where $\mathcal{I}_{t_{\text {tip }}}^{t_{k}}=\left\{t_{i} \in \mathcal{I}: t_{\text {tip }}<t_{i}<=t_{k}\right\}$ is the set of scores of inliers whose parameter value is greater than our current upper limit for the parameter range $t_{\mathrm{tip}}$, and $\sigma$ is a threshold. An analogous check is performed for $t_{k}<t_{\text {base }}$. This condition only allows extensions of the range to inlier positions that are close to the existing model or are not isolated.

\subsection{Experimental Procedure}

Apparatus. A SonixMDP ultrasound console (Ultrasonix Medical Corp., Richmond, Canada) with a tracked (SonixGPS) linear 2D transducer (L14-5/38) was used for imaging. An asymmetric-tip Nitinol needle with outer diameter of 0.8 $\mathrm{mm}$ was inserted along a curved path in ex vivo bovine liver tissue obtained fresh from a local butcher. The needle was connected to a voice coil actuator and attached to a needle steering robot. The needle segmentation method was implemented in $\mathrm{C}++$ and used the Ultrasonix research SDK to receive ultrasound image data $(640 \times 480$ array of RGB pixels) in real time over a network connection. The segmentation software ran on a typical Core i5 processor (Intel Corp., Santa Clara, CA).

Procedure. A reference segmentation was created for comparison by manually identifying the needle in each image, and fitting a 3D polynomial to the identified points. Range was defined based on the extent of the manual points. Nine scans were performed for nine different curved needle paths, with radius of curvature ranging from $30 \mathrm{~cm}$ to $6.8 \mathrm{~cm}$. Ultrasound imaging depth was set to $4 \mathrm{~cm}$, Doppler pulse repetition frequency (PRF) was set to $1.4 \mathrm{kHz}$, and Doppler wall motion filter (WMF) was set between $450 \mathrm{~Hz}$ and $600 \mathrm{~Hz}$ for all tests.

\section{Results}

Across nine tests, the mean error between manual segmentation and the method's results was $0.38 \pm 0.27 \mathrm{~mm}$. The average discrepancy between estimated tip position and manual tip position was $0.71 \pm 0.55 \mathrm{~mm}$. While we do not have an absolute measure of the accuracy of manual segmentation, we show that our method consistently produces results that match manual segmentation.

The robustness and efficiency of the segmentation method result from the use of the Doppler response as a highlighter for B-mode segmentation. This response provides three advantages: (1) an indication of the extents and locations of potential needle cross-sections within a frame, which means the method does not rely 


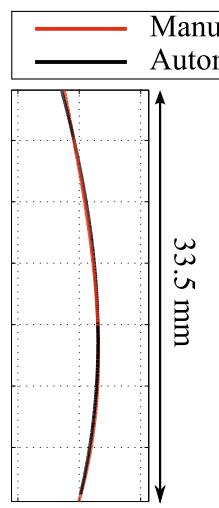

(a)

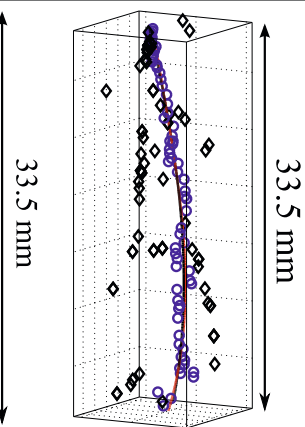

(b)

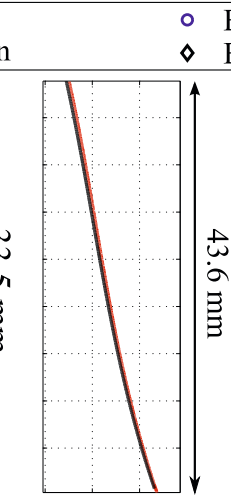

(c)
B-mode Inlier

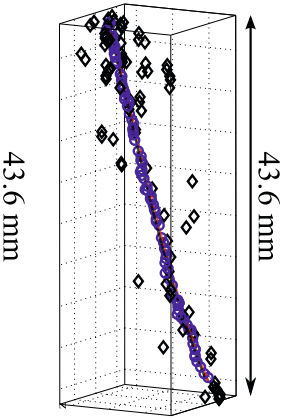

(d)

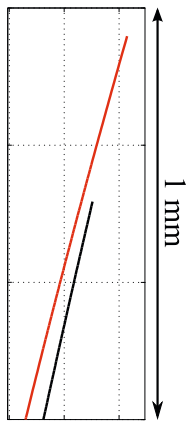

(e)

Fig. 4. Segmentation results (Table 1): (a) Automatic segmentation and reference manual segmentation for Scan 6. (b) Inlier and outlier candidate points for Scan 6. (c) Segmentation output and reference manual segmentation for Scan 3. (d) Inlier and outlier candidate points for Scan 3. (e) Comparison of estimated needle tip position with manual segmentation for Scan 1 . The tip position difference $(0.85 \mathrm{~mm})$ was primarily along the needle's axis.

Table 1. Error and Performance Metrics for each Scan

\begin{tabular}{|l|c|c|c|c|c|c|c|c|c|}
\hline Scan & 1 & 2 & 3 & 4 & 5 & 6 & 7 & 8 & 9 \\
\hline \hline Tip Error $(\mathrm{mm})$ & 0.85 & 0.25 & 0.84 & 1.78 & 0.14 & 1.35 & 0.43 & 0.38 & 0.40 \\
\hline Mean Seg. Error (mm) & 0.370 & 0.300 & 0.372 & 0.308 & 0.394 & 0.441 & 0.316 & 0.438 & 0.504 \\
\hline Seg. Error Std. Dev. (mm) & 0.202 & 0.133 & 0.171 & 0.280 & 0.249 & 0.238 & 0.189 & 0.316 & 0.611 \\
\hline Exec. Time (ms) & 5.16 & 6.14 & 6.89 & 7.58 & 6.64 & 5.97 & 7.12 & 7.93 & 9.26 \\
\hline Needle Radius (cm) & 33.59 & 20.62 & 23.48 & 61.33 & 20.17 & 6.98 & 8.22 & 13.99 & 9.69 \\
\hline
\end{tabular}

on the needle being the most echoic structure in the frame, (2) a rough first model of 3D needle shape for outlier rejection, and (3) an indication of when the transducer has moved past the needle during a scan. An important application of this work is guidance of a needle steering robot. To generate nearly real-time feedback of needle tip pose and position with this method, an operator or robot must follow the movement of the needle as it is inserted so that the needle-tip is contained within the ultrasound image plane as often as possible.

\section{Conclusion}

We described a method for segmenting curved needles based on a fusion of Doppler and B-mode ultrasound data. This method is more accurate, more robust, and faster than segmentation based on only one modality. Experiments demonstrated that this method results in an error on the order of $0.5 \mathrm{~mm}$ along the needle curve and $1 \mathrm{~mm}$ at the tip in ex vivo bovine tissue when compared to manual segmentation. This method executes in less than $10 \mathrm{~ms}$ per time step 
on a standard processor. In future work, we will test the method in in vivo animal experiments, where organ movement and blood-flow may generate Doppler responses that compete with the needle vibration response.

\section{References}

1. Aboofazeli, M., Abolmaesumi, P., Mousavi, P., Fichtinger, G.: A new scheme for curved needle segmentation in three-dimensional ultrasound images. In: IEEE Int. Symp. Biomedical Imaging: Nano to Macro, pp. 1067-1070 (2009)

2. Adebar, T.K., Okamura, A.M.: 3D segmentation of curved needles using doppler ultrasound and vibration. In: Barratt, D., Cotin, S., Fichtinger, G., Jannin, P., Navab, N. (eds.) IPCAI 2013. LNCS, vol. 7915, pp. 61-70. Springer, Heidelberg (2013)

3. Cheung, S., Rohling, R.: Enhancement of needle visibility in ultrasound-guided percutaneous procedures. Ultrasound Med. Biol. 30(5), 617-624 (2004)

4. Ding, M., Cardinal, H.N., Fenster, A.: Automatic needle segmentation in 3D ultrasound images using two orthogonal 2D image projections. Med. Phys. 30(2), 222-234 (2003)

5. Ding, M., Fenster, A.: A real-time biopsy needle segmentation technique using hough transform. Med. Phys. 30(8), 2222-2233 (2003)

6. Fronheiser, M.P., Idriss, S.F., Wolf, P.D., Smith, S.W.: Vibrating interventional device detection using real-time 3 -D color doppler. IEEE Trans. Ultrason. Ferroelectr. Freq. Control 55(6), 1355-1362 (2008)

7. Harmat, A., Rohling, R.N., Salcudean, S.E.: Needle tip localization using stylet vibration. Ultrasound Med. Biol. 32(9), 1339-1348 (2006)

8. Holen, J., Waag, R.C., Gramiak, R.: Improved needle-tip visualization by color Doppler sonography. Am. J. Roentgenol. 156, 401-402 (1985)

9. Klein, S.M., Fronheiser, M.P., Reach, J., Nielsen, K.C., Smith, S.W.: Piezoelectric vibrating needle and catheter for enhancing ultrasound-guided peripheral nerve blocks. Anesth. Analg. 105(6), 1858-1860 (2007)

10. Mung, J., Vignon, F., Jain, A.: A non-disruptive technology for robust 3D tool tracking for ultrasound-guided interventions. In: Fichtinger, G., Martel, A., Peters, T. (eds.) MICCAI 2011, Part I. LNCS, vol. 6891, pp. 153-160. Springer, Heidelberg (2011)

11. Neshat, H.R.S., Patel, R.V.: Real-time parametric curved needle segmentation in 3D ultrasound images. In: IEEE RAS EMBS Int. Conf. Biomedical Robotics Biomechatronics, pp. 670-675 (2008)

12. Okazawa, S.H., Ebrahimi, R., Chuang, J., Rohling, R.N., Salcudean, S.E.: Methods for segmenting curved needles in ultrasound images. Med. Im. Anal. 10(3), 330-342 (2006)

13. Otsu, N.: A threshold selection method from gray-level histograms. Automatica 11, 23-27 (1975)

14. Uhercík, M., Kybic, J., Liebgott, H., Cachard, C.: Model fitting using RANSAC for surgical tool localization in 3D ultrasound images. IEEE Trans. Biomed. Eng. 57(8), 1907-1916 (2010)

15. Zhou, H., Qiu, W., Ding, M., Zhang, S.: Automatic needle segmentation in 3d ultrasound images using 3d improved hough transform. In: Miga, M., Cleary, K. (eds.) SPIE Proceedings 2008, vol. 6918, pp. 691821-691821-9 (2008) 